\title{
Viewing Experience of Augmented Reality Objects as Ambient Media - A Comparison of Multimedia Devices
}

\author{
Ilhan Aslan, Chi Tai Dang, Björn Petrak, Michael Dietz, Michael Filipenko, \\ and Elisabeth André \\ Human-Centered Multimedia Lab, Augsburg University, Germany \{aslan, dang, \\ petrak, dietz, filipenko, andre\}@hcm-lab.de
}

\begin{abstract}
Augmented reality objects as ambient media (i.e., media embedded in everyday contexts) are being deployed in diverse contexts, such as home, mobile, and work. Because media experience is mediated by the medium, the viewing experience of augmented reality objects should depend on the type of viewing device. In this paper, we report on a user study with 18 participants and three state-of-the-art devices (i.e., HoloLens, iPad Pro tablet, and iPhone X smartphone), comparing the influence of these mobile viewing devices on feelings of presence and workload during ambient media consumption. Study results include a significant main effect of device on participants ratings of Possibility to examine an AR object, which demonstrates that ambient media consumption experiences are determined by the choice of viewing device.
\end{abstract}

Keywords: Augmented Reality · User Experience · Multimodality

\section{Introduction}

There is an ongoing hype in augmented reality (AR) research and near future visions depicted in contemporary movies, which suggest that AR objects will increasingly become part of our everyday lives and serve as ambient media. To compare how different mobile devices impact users' viewing experience of AR Objects we have conducted an empirical study with 18 participants. Our assumption was that different classes of mobile devices (i.e., head-mounted, tablet, smartphone) may influence viewers' feelings of presence and workload when spatio-visually exploring an AR object. To this end, we report and discuss participants' self-reports on feelings of workload and presence, including their dimensions Possibility to examine and Quality of interface, which show that today's mobile devices seem to provide a different AR object viewing experience in a simple viewing task than the HoloLens as a state-of-the-art head-mounted display. Overall our research demonstrates that the choice of AR Object viewing device significantly matters considering the viewing experiences, and moreover our research describes specific differences in todays' state of the art viewing devices with a focus on feelings of presence and workload. 

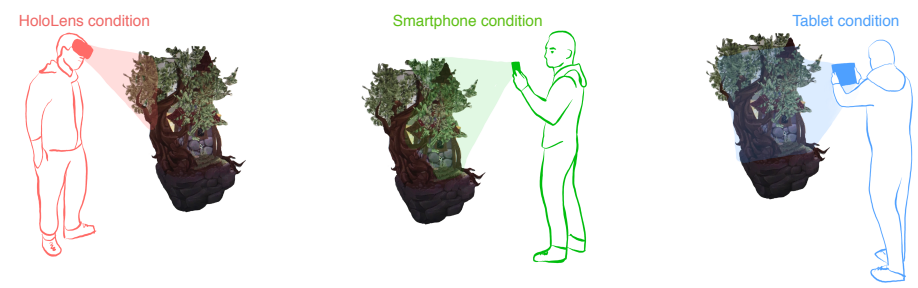

Fig. 1. Images of the treehouse AR object and sketches of the viewing conditions employed to explore the effect of viewing medium on feelings of presence and workload.

\section{User Study}

The overall goal of the user study was to explore differences in feelings of presence and workload associated with different classes of mobile devices used as mediums/modalities to view AR objects. We targeted three types of state of the art mobile devices: (i) smartphone (ii) tablet, and (iii) head-mounted mixed reality glass, with smartphone and tablet being video-see-through devices in different sizes and the head-mounted device (i.e., HoloLens) being a binocular optical-see-through device (see Figure 1). Consequently, our hypothesis was that the type of viewing device effects feelings of presence and workload during an AR object viewing task. To be gender inclusive and because some related work suggests potential gender differences in spatio-cognitive abilities (e.g., $[1,2,4]$ ) we choose to gender-balance our study.

\subsection{Participants, Apparatus and Procedure}

We recruited 18 participants (aged between 18-40, 9f and $9 \mathrm{~m}$ ) at the university campus. All participants have reported to have no color vision deficiency and were "tech-savy" and accustomed to using mobile devices. Ten of these participants reported to already have varying levels of experience with augmented reality. The study was conducted at an open space inside the university building. The study space was about 40 square meters and away from potential by-passers. The three devices, which we utilized in the user study as viewing mediums were (i) an iPhone X smartphone, (ii) a first generation iPad Pro tablet, and (iii) a first generation Microsoft's HoloLens. We chose these devices because we believe that they are archetypical and therefore results are likely to be ecologically valid.

For each of the devices an application was developed, which calculates the exact position to place the AR object based on augmented reality software, and thus allows users to visually perceive the AR object as an overlay to the real world (image). The applications also enable users to implicitly (and explicitly) interact with AR objects by moving around the AR object or holding the viewing medium differently. For the iOS devices (i.e., the iPad and iPhone) we used Apple's ARKit ${ }^{1}$ and its plane detection to find a surface, allowing to place the AR

\footnotetext{
${ }^{1}$ https://developer.apple.com/arkit/
} 
object at the desired location. The application for the HoloLens was developed using Unity ${ }^{2}$ and Microsoft's HoloToolkit.

For the purpose of self-reporting feelings of presence, we used the Presence Questionnaire (PQ), which is adapted from Witmer and Singer [5] and revised by the UQO Cyberpsychology Lab (2004). The PQ measures overall presence and consists of five sub-scales: realism, possibility to act, quality of interface, possibility to examine, and self-evaluation of performance, which combined measure overall presence. In addition, we used the NASA-TLX questionnaire [3] to explore how the viewing medium may change feelings of perceived workload (i.e., mental workload, physical workload, temporal workload, performance, effort, frustration, and overall workload). A semi-structured interview was also conducted at the end with each participant.

The user study started with welcoming each participant and handing them a written short description of the study procedure, which stated that participants would receive three augmented reality devices, one after another, with which they would be able to observe an AR object positioned at the center of the study space. Participants were informed that their task was to view the AR object for 3 minutes and try to remember as many details of the AR object as possible since we would ask them a question after each session about a detail of the AR object. At the end of each session participants were asked to provide self-reports on the viewing experience associated with the specific device based on the PQ and NASA-TLX questionnaire. We used counterbalancing (levels of device and gender) to ensure validity of our study. To ensure participants would view the AR object in each of the three repetitions carefully, they were informed that they would be asked a different detail of the AR object after each session. We asked for example "Which color did the door knob have?". Each participant completed the study in about 45 minutes.

\subsection{Results}

In terms of viewing device preferences, $9(2 \mathrm{f}, 7 \mathrm{~m})$ participants preferred the tablet, 5 (5f) participants preferred the HoloLens and $4(2 \mathrm{f}, 2 \mathrm{~m})$ preferred the smartphone. The frequency plots in Figure 2 provide a descriptive overview of the collected data, considering feelings of workload and presence. Considering the mean ratings for presence, the tablet has received the highest mean scores on all dimensions. An exception are ratings of female participants for the dimension Realism, who have provided very similar mean ratings for the HoloLens and the tablet. Besides this exception the HoloLens device has received the lowest mean scores in our sample data. Participants seem to have associated low workload with all devices and the viewing tasks. The Performance dimension of the NASA-TLX questionnaire seems to be an exception. Participants provided higher ratings for the Performance dimension, which measures how much "performance demand" participants felt when using a specific device to complete the viewing task.

\footnotetext{
2 https://unity3d.com
} 


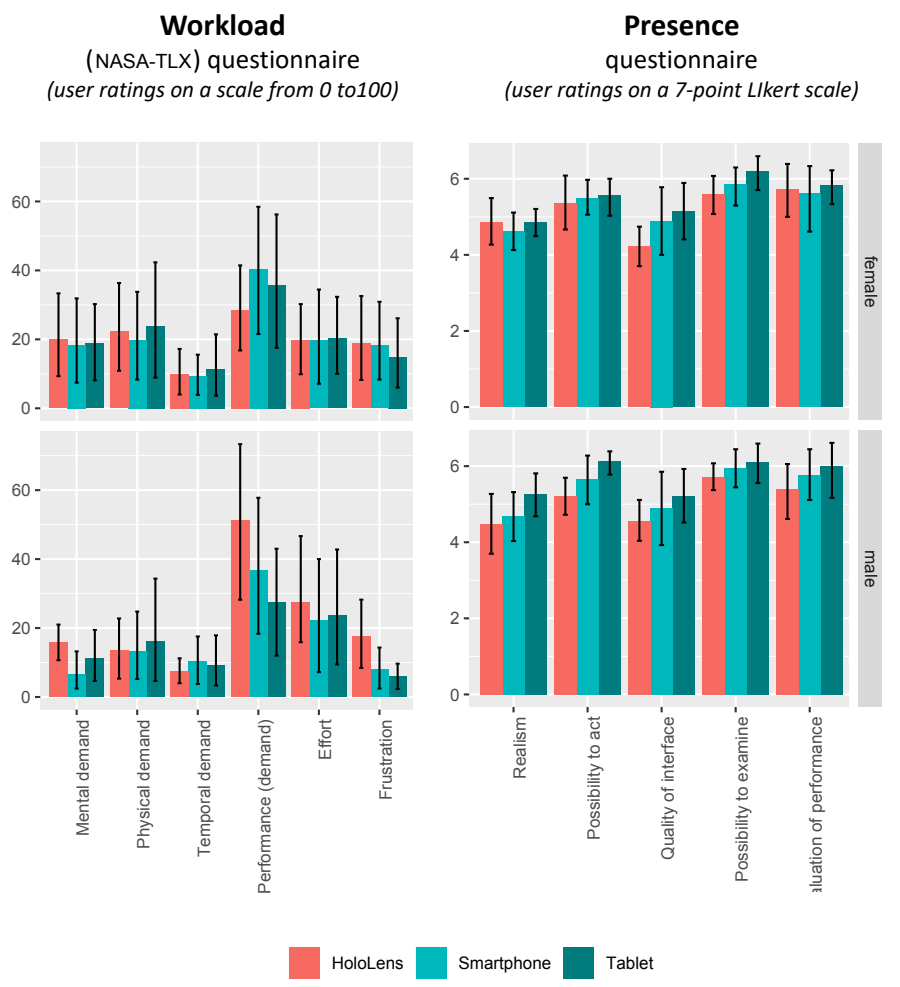

Fig. 2. Overview of the data collected with the workload questionnaire and the presence questionnaire. Descriptive statistics (i.e., mean values and confidence intervals) are presented for all ratings for sub-scales of presence and workload separated by the levels of the two independent variables device and gender. Error bars denote $95 \%$ CI.

Overall, the plots in Figure 2 show that the HoloLens received worse mean scores compared to the other viewing devices from males for both feelings of workload and presence, which could be part of the reason why none of the male participants preferred the HoloLens overall for viewing AR objects.

Statistical Analysis Table 1 depicts the results of the analysis for which we used ARTool [6]. In order to save space in the table we present only test results, which resulted in p-values below 0.1 . While there are some measurements that resulted in a p-value below the 0.1 threshold, including a potential main effect of device on overall presence $(\mathrm{F}=2.63, \mathrm{p}=0.087)$ only two measurements are statistically significant (i.e., have p-values below 0.05 ). We found a significant main effect of device on possibility to exam $(\mathrm{F}=3.86, \mathrm{p}=0.031)$ and a significant interaction between gender and device considering performance (demand). Post-hoc cross-factor comparisons highlight that this interaction is due to significant dif- 


\begin{tabular}{|l|l|l|l|}
\hline Effect of: & Effect on: & $F$ and $p$ & Post-hoc comp. \\
\hline \hline Device & Overall presence & $\mathrm{F}=2.63$ & \\
& (perceived presence) & $p=.087$. & \\
\hline \hline Device & Quality of interface & $\mathrm{F}=3.07$ & \\
& (perceived presence) & $p=.060$. & \\
\hline \hline Device & Possibility to examine & $\mathrm{F}=3.86$ & \\
& (perceived presence) & $p=.031^{*}$ & \\
\hline \hline Device & Mental demand & $\mathrm{F}=2.68$ & \\
& (perceived workload) & $p=.083$. & \\
\hline \hline Device $*$ & Performance demand & $\mathrm{F}=3.67$ & $\mathrm{~T}-\mathrm{H}: \mathrm{F}-\mathrm{M}(\mathrm{p}=.03 *) ;$ \\
Gender & (perceived workload) & $p=.036 *$ & S-H:F-M $(\mathrm{p}=.08)$. \\
\hline \hline Device & Effort & $\mathrm{F}=2.52$ & \\
& (perceived workload) & $p=.095$. & \\
\hline
\end{tabular}

Table 1. Overview of results of statistical tests (based on the ARTool [6]) over all participants, considering the overall effect of within-subjects variable Device (i.e., HoloLens, Smartphone, and Tablet), between-subjects variable Gender (i.e., male and female), and Interactions of Device and Gender on measures for perceived presence and workload. Abbreviations used for reporting pairwise comparison: Tablet-HoloLens (T-H), Tablet-Smartphone (T-S), and Smartphone-HoloLens (S-H). F-M is an abbreviation for Female-Male.

ferences between HoloLens and tablet and HoloLens and smartphone. If we try to put the statistics in words, it would mean that the average difference in "performance demand" perceived by male participants between the HoloLens and the other two viewing mediums was significantly opposite to female participants's perception of the same conditions.

Analysis of qualitative data In the following, we list results of observations of participants' behaviors during the viewing task and results of the semi-structured interviews which were conducted at the end of each study with each participant. HoloLens: When using the HoloLens, participants tended to keep a larger distance to the AR object, which resulted in participants moving at the edge of the study space mostly around the AR object and occasionally moving towards and away from the AR object. Arguments of participants who preferred the HoloLens were focused on reasons why the HoloLens provided a more natural and realistic experience. Participants argued, for example that "it felt more natural" and that it is "most realistic because one does not have to look through a screen". Furthermore, participants stated "more realistic especially when one has their hands free" and more realistic because "the AR object moves [in synchrony] with head movements".

Smartphone and tablet: When using the smartphone or the tablet participants not only moved around the AR object but additionally used the possibil- 
ity to rotate or move the viewing medium without changing their own position. Most of the time participants used the handhelds in portrait format, while in the HoloLens' orientation is landscape and fix. Furthermore, handhelds allowed participants to view the AR object from above, below and from the side without always having to move around and instead adjusting their screens position by moving the screen closer, away, to the side, etc. The smartphone was usually held with one hand only and the tablet was always held with both hands. Participants who preferred the tablet focused on benefits of the larger screen, arguing "the viewing angle of the HoloLens and the smartphone is too small', "most details visible' and "HoloLens is too demanding and has a too small view, and the smartphone is too small'. Participants who preferred the smartphone focused on its "better overall usability", arguing that "it is easiest to change the viewing angle" or how it is "better to handle, not as heavy as the tablet", and while it is "small but enough is visible".

\section{Conclusion}

We have reported on an empirical study with users, exploring the influence of state-of-the-art (and off-the-shelf) augmented reality devices on viewing experience. We have provided an analysis of data associated with the feeling of presence and workload, showing for example that today's handheld devices are perceived as providing significantly more Possibility to examine an AR object. We hope the presented research is timely and many fellow researchers will benefit from a detailed analysis of how viewing devices effect AR object viewing experiences.

\section{Acknowledgments}

This research was partly funded by the BMBF (Ministry of Education and Research) in the DIGISTA project (no. 01UO1820A).

\section{References}

1. Coluccia, E., Louse, G.: Gender differences in spatial orientation: A review. Journal of environmental psychology 24(3), 329-340 (2004)

2. Geary, D.C.: Sexual selection and sex differences in spatial cognition. Learning and Individual Differences 7(4), 289 - 301 (1995)

3. Hart, S.G., Staveland, L.E.: Development of nasa-tlx (task load index): Results of empirical and theoretical research. In: Advances in psychology, vol. 52, pp. 139-183. Elsevier (1988)

4. Linn, M.C., Petersen, A.C.: Emergence and characterization of sex differences in spatial ability: A meta-analysis. Child development pp. 1479-1498 (1985)

5. Witmer, B.G., Singer, M.J.: Measuring presence in virtual environments: A presence questionnaire. Presence 7(3), 225-240 (1998)

6. Wobbrock, J.O., Findlater, L., Gergle, D., Higgins, J.J.: The aligned rank transform for nonparametric factorial analyses using only anova procedures. In: Proceedings of the SIGCHI Conference on Human Factors in Computing Systems. pp. 143-146. CHI '11, ACM, New York, NY, USA (2011) 